\title{
Control of cell-cell forces and collective cell dynamics by the intercellular adhesome
}

Elsa Bazellières ${ }^{1}$, Vito Conte ${ }^{1}$, Alberto Elosegui-Artola ${ }^{1}$, Xavier Serra-Picamal ${ }^{1}$, María

Bintanel-Morcillo ${ }^{1}$, Pere Roca-Cusachs ${ }^{1,2}$, José Muñoz ${ }^{3}$, Marta Sales-Pardo ${ }^{4}$, Roger

Guimerà $^{4,5}$, Xavier Trepat ${ }^{1,2,5^{*}}$

${ }^{1}$ Institute for Bioengineering of Catalonia, Barcelona, Spain.

${ }^{2}$ Unitat de Biofísica i Bioenginyeria, Facultat de Medicina, Universitat de Barcelona, and CIBERES, Spain

${ }^{3}$ Laboratori de Càlcul Numèric, Department of Applied Mathematics III, Universitat Politècnica de Catalunya, Barcelona, Spain.

${ }^{4}$ Departament d'Enginyeria Química, Universitat Rovira i Virgili, Av. Països Catalans 26, Tarragona 43007, Catalonia, Spain

${ }^{5}$ Institució Catalana de Recerca i Estudis Avançats (ICREA), Barcelona, Spain.

${ }^{*}$ Corresponding author:

Prof. Xavier Trepat,

Institute for Bioengineering of Catalonia

C/ Baldiri Reixac 15-21

Barcelona 08028

Spain.

Tel: +34934020265

Email: xtrepat@ub.edu 


\section{Summary}

Collective migration and remodeling of epithelial sheets enable fundamental processes in morphogenesis, tissue repair, and cancer invasion. The dynamics of these collective processes is critically influenced by intercellular forces but underlying control mechanisms remain unknown. Here we provide a systematic study of the interplay between cell-cell adhesion proteins, cell-cell forces, and epithelial tissue dynamics. We show that collective cellular responses to systematic perturbations of the intercellular adhesome conform to three distinct mechanical phenotypes. These phenotypes are controlled by different molecular modules and characterized by distinct relationships between cellular kinematics and intercellular forces. We show that these forces and their rates can be predicted by the concentrations of cadherins and catenins. Unexpectedly, we identified distinct mechanical roles for P-cadherin and E-cadherin; while P-cadherin predicts overall levels of intercellular force, E-cadherin predicts the rate at which intercellular force builds up. Together, our data unveil different strategies by which cell-cell adhesion proteins control cell-cell forces and epithelial dynamics.

\section{Highlights}

- Systematic knock down of intercellular adhesion proteins results in three mechanical phenotypes.

- Concentrations of cadherins and catenins predict intercellular forces and their time evolution.

- P-cadherin predicts intercellular tension while E-cadherin predicts its buildup rate.

- Force applied through P-cadherin or E-cadherin triggers different feedback loops. 


\section{Introduction}

The homeostasis of epithelial tissues and its disruption during disease are enabled by collective cellular processes such as growth, migration, and remodeling (Friedl and Gilmour, 2009; Heisenberg and Bellaiche, 2013; Weber et al., 2012). Each of these collective processes has long been known to be critically influenced by cell-cell adhesion complexes. To a significant extent, this critical influence has its mechanistic origin in the ability of cell-cell adhesions to transmit physical forces (Leckband et al., 2011; Budnar and Yap, 2013). During collective epithelial migration, for example, a broad variety of cell types migrate along the direction of maximal intercellular tension (Tambe et al., 2011). By contrast, during neural crest morphogenesis, a transient exchange of intercellular force appears to elicit repulsive signals between clusters of different cell types (Theveneau et al., 2013). Forces transmitted through cell-cell junctions have also been shown to orient cell shape and division and thus contribute to tissue growth and anisotropy (Bosveld et al., 2012; Campinho et al., 2013; Legoff et al., 2013). Finally, cell-cell junctions allow for the propagation of mechanical oscillations and waves across long distances, thus enabling the transmission of mechanical information across tissues (Martin et al., 2009; Solon et al., 2009; Blanchard et al., 2010; Rauzi et al., 2010; SerraPicamal et al., 2012).

Force transmission through intercellular junctions is traditionally attributed to adherens junctions, which provide a physical connection between the actomyosin cytoskeleton and transmembrane proteins of the cadherin superfamily (Steinberg, 2005; Borghi et al., 2012; Foty and ; Maitre and Heisenberg, 2013; Zaidel-Bar, 2013). The existence of this physical connection is supported by an extensive body of experimental evidence but the identity of the molecules that transmit physical forces remains controversial. For example, epithelial cells often express several cadherin isoforms (Nieman et al., 1999; Ribeiro et al., 2010), including E-cadherin, N-cadherin, and P-cadherin, but the extent to which each of these classical cadherins is involved in force transmission is unknown (Tabdili et al., 2012). Moreover, the network of proteins that provides a direct physical connection between classical cadherins and the cytoskeleton is also incomplete. $\beta$-catenin and $\alpha$-catenin have long been known to be involved in junction stability and tissue cohesiveness, but their ability to provide a direct link between cadherins and the cytoskeleton is currently unclear (Drees et al., 2005; Yamada et al., 2005). Consequently, other proteins such as ZO-1, vinculin, and lima have been proposed to provide the missing link for force transmission (Abe and Takeichi, 2008; Zaidel-Bar, 2013).

Besides adherens junctions, the actomyosin cytoskeleton of adjacent cells is also connected through tight junctions, which comprise transmembrane receptors such as claudins, occludin, and JAM-A, as well as intracellular linkers such as ZO-1, ZO-2, and ZO-3 (Runkle and Mu, 2013). Tight junctions are typically associated with epithelial sealing rather than force transmission, but no experimental evidence has so far ruled out the ability of tight junctions to resist and transmit physical forces. In addition to the actin cytoskeleton, forces within epithelial sheets have also been proposed to be transmitted by intermediate filaments within cells and by desmosomes between cells (Herrmann et al., 2007; Wang and Stamenovic, 2000; Harris et al., 2012). Finally, gap junctions, which 
play a major role in controlling the intercellular transit of ions and small solutes between cells, have been shown to be mechanosensitive (Bao et al., 2004), thus raising the possibility that they might play a mechanical role in epithelial tissue dynamics.

Here we used micropatterned sheets of epithelial cells as a model system to study the interplay between intercellular adhesion proteins, physical forces, and tissue dynamics. We designed a minimal custom library of validated siRNAs targeting the main molecular components of the intercellular adhesome. For each siRNA perturbation we measured cellular velocities and deformation rates, as well as inter-, intra-, and extra-cellular forces. Using unsupervised clustering analysis, we identified systematic relationships between these physical properties and molecular control modules within the adhesome. Using a cross-validation analysis we established the ability of intercellular adhesion proteins to quantitatively predict tissue dynamics.

\section{Results}

\section{Intercellular cohesiveness increases with monolayer expansion}

We developed an assay to measure in parallel the epithelial dynamics of multiple expanding monolayers (Figure S1). Using soft lithography, thin PDMS membranes with a rectangular opening were fabricated and deposited on top of a collagen I-coated polyacrylamide gel substrate (Poujade et al., 2007; Serra-Picamal et al., 2012). We then seeded normal breast epithelial cells (MCF10A) and allowed them to adhere and spread until they formed a confluent monolayer. After $5 \mathrm{~h}$ of culture, F-actin was largely cortical but the monolayer was not cohesive; cadherins, catenins, and ZO-1 were either fully cytoplasmic or weakly localized at the lateral cell-cell contact areas (Figure 1A,C).

Upon lifting the PDMS membrane, the monolayer expanded toward the freely available gel surface and, after 8 hours of migration, its area had increased by $\sim 30 \%$ (Figure 1E,F,M, Movie S1). At this point, cadherins, catenins, and ZO-1 progressively accumulated at the cell cortex, and this accumulation was more pronounced in the central region of the monolayer (Figure 1B,D). Thus, as the monolayer expanded it increased its structural cohesiveness through recruitment of cell-cell adhesion proteins to the cell cortex.

\section{As the monolayer increases its cohesiveness, cell migration slows down and intercellular forces buildup}

We next studied how the increase in monolayer cohesiveness was paralleled by changes in physical properties of the constituent cells, including cellular velocities, inter- and intra-cellular forces, and traction forces at the cell-substrate interface. To map cell velocities during monolayer expansion we used particle imaging velocimetry (PIV) (Serra-Picamal et al., 2012). As shown previously in different cell types, measured velocity fields showed large spatial heterogeneities that spanned several cell diameters (Figure1G,H, Movie S2) (Tambe et al., 2011; Serra-Picamal et al., 2012). Despite these 
large fluctuations, cells moved predominantly away from the initial pattern and, as the monolayer expanded, the average cell velocity decreased (Figure $1 \mathrm{~N}$ ).

Simultaneously with cell velocities we measured traction forces exerted by cells on the underlying substrate. From the very onset of monolayer expansion, traction forces exhibited a punctate spatial distribution characterized by rapid fluctuations in magnitude and direction (Figure 1I,J, Movie S2). The magnitude of these fluctuations was similar across the monolayer, thus showing that monolayer expansion involved the active contribution of cells from the leading edge and behind it. To assess the total traction exerted by cells in the monolayer we computed the strain energy density $(U)$ transferred by cells to the gel. $U$ increased with time and tended to saturate after 300 minutes (Figure 1O), indicating that cells approached but never reached a mechanical steady state.

We next asked whether the accumulation of adhesion proteins at intercellular contacts was paralleled by an increase in cell-cell forces. To address this question we used Monolayer Stress Microscoscopy (MSM), which computes the state of mechanical stress (force per unit cross-sectional area) everywhere within the cell sheet (Tambe et al., 2011; Hur et al., 2012; Serra-Picamal et al., 2012; Trepat and Fredberg, 2012; Tambe et al., 2013). For simplicity, we mainly focused on $\sigma_{x x}$, the stress component in the direction of expansion of the monolayer, and referred to it indistinctly as monolayer tension or intercellular tension (Figure S2 for other stress components). Monolayer tension displayed well-known features of expanding epithelial sheets including dynamic heterogeneities and supracellular fluctuations (Figure 1K,L, Movie S2) (Tambe et al., 2011; Hur et al., 2012; Serra-Picamal et al., 2012). Similarly to the case of traction forces, all components of the stress tensor increased monotonically with time and tended to plateau as the expansion slowed down (Figure 1P).

Taken together, our structural and mechanical data put forward the following scenario. As the monolayer expands, it matures its intercellular adhesions with a cortical recruitment of cadherins, catenins, and ZO-1. These structural changes are paralleled by a progressive decrease in cell velocity, and a pronounced increase in cell-substrate forces and intercellular tension.

\section{Downregulation of cell-cell adhesion proteins alters cell velocities, cell tractions, and intercellular tension}

We next sought to identify what proteins are involved in the regulation and transmission of monolayer tension. To do so, we designed a minimal siRNA library to selectively knock down the main molecular players of the intercellular adhesome (Figure S3). Specifically, we targeted key transmembrane proteins associated with adherens junctions, tight junctions, desmosomes, and gap junctions. In addition, we targeted a subset of cytoplasmic proteins that have been shown or proposed to be involved in linking these transmembrane proteins to the actin and intermediate filament cytoskeleton.

To effectively knockdown genes coding for intercellular adhesion proteins we transfected cells with a pool of 3 distinct siRNAs and carried out monolayer expansion experiments 5 
days later. All siRNA pools efficiently down-regulated their respective target genes with an average mRNA knockdown of $81 \% \pm 11 \%$ (mean \pm SEM) (Figure S4A).

The mechanical responses to siRNAs were diverse in terms of cell velocities, cellsubstrate tractions, and intercellular tensions. Representative maps of $v_{x}, T_{x}$ and $\sigma_{x x}$ for a subset of siRNAs are shown in Fig. 2 (see also Figures S5 to S10 for a complete set of representative experiments). These maps confirm previous studies on the same cell type showing that down-regulation of E-cadherin or N-cadherin has no impact on cellular velocities (Figure 2B, Figure S5) (Simpson et al., 2008; Kumper and Ridley, 2010). Our data show, further, that loss of function of these cadherins does not influence the transmission of intercellular tension (Figure 2B, Figure S5, Movie S3). By contrast, knocking down P-cadherin led to monolayer dissociation, an increase in cell velocity and a sharp drop in monolayer tension (Figure 2C). Similarly, downregulation of catenins led to faster monolayer expansion and lower intercellular tension (Figure 2D-F, Figure S6). Knock down of lima1 and Drrl did not impact significantly monolayer dynamics (Figure S7). Together, these data suggest that P-cadherin, but not E-cadherin or N-cadherin, associates with catenins to mediate intercellular force transmission in MCF10A cells.

siRNAs targeting desmosomes, tight junctions, and gap junctions also altered cellular velocities, tractions, and tensions (Figure 2G-L, Figures S8-S10). Downregulation of tight junction proteins ZO-1 and ZO-3, which are traditionally associated with epithelial sealing but not with force transmission, led to marked changes in monolayer mechanics (Figure 2I,J). Interestingly, while knockdown of ZO-1 caused an increase in monolayer tension, knockdown of ZO-3 had the opposite effect. Depletion of tight junction protein JAM-A caused a large drop in cell-substrate tractions but had only a mild effect on intercellular tension (Figure $2 \mathrm{H}$ ). Downregulation of desmosomal proteins caused minor changes in monolayer forces and kinematics with the exception of Dsc3, which slowed down cell migration (Figure 2K, Figure S10). Finally, siRNAs targeting gap junction protein $\mathrm{Cx} 43$ caused a weak decrease in traction forces and intercellular tension (Figure $2 \mathrm{~L}$ ). Thus, proteins associated with junctional complexes other than adherens junctions also appear to be involved in the regulation of monolayer dynamics.

We next focused on the time evolution of monolayer dynamics in response to siRNAs. For each time point, we averaged maps of each physical property across space and over all experimental repeats. The time evolution of these averages showed that the global trends of control cases were generally conserved under siRNA perturbations (Figure 2M$\mathrm{P}$, Figure S11). For example, all monolayers expanded, though at a different rate and to a different extent (Figure 2M). Likewise, all monolayers exhibited increases in their traction forces (Figure 2O). By contrast, some physical properties were affected both quantitatively and qualitatively. For example, some siRNAs altered the tendency of cells to slow down with time and caused transient increases in cellular velocity (e.g. sip120) (Figure 2N). Similarly, the general tendency of intercellular forces to increase with monolayer expansion was disrupted by a number siRNAs -e.g. sip120, si $\beta$ Cat, and siPCad-, which prevented cells from building up intercellular tension (Figure 2P). Taken together, these data indicate that proteins from distinct junctional complexes alter 
monolayer kinematics and forces, as well as the time evolution of these physical properties.

\section{Forces and kinematics are anti-correlated}

Our siRNA experiments provided us with an extensive body of data to explore the distinct dynamic regimes that cells within the monolayer access collectively. To study these mechanical regimes systematically, we first asked whether physical properties that define tissue dynamics are linked through universal relationships or whether they are generally uncorrelated. To address this question, we begun by reducing the number of measured physical properties to a minimal set of properties that fully captured the diversity of the observed mechanical phenotypes. We selected the following properties, averaged over time, space, and experimental repeats: cell velocity $v_{x}$, cell deformation rate $\varepsilon_{x x}$, increase in monolayer area $\Delta A$, strain energy transferred by cells to the substrate $U$, and maximum shear stress $\sigma_{s}$ (Experimental Procedures). In addition, to capture monolayer tension to first order in time we considered the average value of $\sigma_{x x}$ at the end of the experiment $\left(\sigma_{x x}^{f}\right)$ and the average slope of its time evolution ( $\left.\sigma_{x x}^{*}\right)$. In this way, we were able to summarize our data in a $m \times n$ matrix comprising $m$ siRNA perturbations, and $n$ physical properties $(m=21, n=7)$. From this data matrix, we computed a matrix $Z$, in which each element $Z_{i j}$ (element located on row $i$ and column $j$ ) contains the $z$-score of the physical property $j$ in response to a siRNA perturbation $i$ (Figure 3A) (see Experimental Procedures for a description of z-score calculations).

When then computed a cross-correlation between z-scores of each possible pair of physical variables (i.e., correlations between columns in Figure 3A). Among the $m$ siRNA perturbations, we considered only the subset of $q$ perturbations having at least one physical property that differed from the control by more than two standard deviations $(q=12)$. Correlation analysis yielded a $n \times n$ symmetric matrix $C p$, where each element $C p_{i j}$ indicates the correlation between physical properties $i$ and $j$ (Figure 3B). We then used an unsupervised algorithm (Sales-Pardo et al., 2007) to cluster together the physical properties with highest correlation.

Our analysis unveiled two clear clusters of highly correlated physical properties (Figure 3B). The first cluster comprises physical properties that describe monolayer kinematics, namely, velocity, expansion, and deformation rate. The second cluster comprises physical variables that describe monolayer forces, namely, the strain energy density, the maximum shear stress, monolayer tension at the end of the experiment and the buildup rate of monolayer tension. Some correlations within each of the clusters were consistent with expectations based on current knowledge of monolayer dynamics. For example, monolayer expansion is expected to be greatest if cells are able to move faster and deform faster, and this was seen to be the case. Other correlations were quite unexpected, however. For example, traction forces were highly correlated with the maximum shear stress in the monolayer but weekly correlated with monolayer tension. Remarkably, the two clusters of physical properties were anti-correlated; as traction forces increased and intercellular tension built up, cells tended to slow down their migration and deformation rates. 


\section{A purely physical readout is sufficient to identify and sort proteins that comprise distinct intercellular complexes}

We next asked what siRNA perturbations had a similar impact on physical properties. To address this question, we computed correlations between pairs of siRNA perturbations (i.e. correlations between rows in Figure 3A). The resulting correlation matrix is a $q \times q$ symmetric matrix $C s$, where each element $C s_{i j}$ indicates the correlation between siRNA pertubations $i$ and $j$ (Figure 3C).

Our unsupervised algorithm identified three clusters of siRNAs as regards their impact on physical properties (Figure 3C). The first cluster includes only the siRNAs targeting ZO1 , which showed a correlation profile that was clearly distinct from any other siRNA perturbation. The second cluster comprises siRNAs targeting all adherens junction proteins as well as the tight junction protein occludin. The third cluster includes siRNAs targeting proteins from diverse intercellular complexes including tight junction proteins Cldn8, ZO-3 and JAM-A, gap junction protein Cx43, and desmosomal protein Dsc3.

The identification of these three clusters is remarkable for two main reasons. First, the presence of all adherens junction proteins into one single cluster shows that a purely physical readout is sufficient to sort the proteins that form a specific molecular complex from those that do not. Second, the functional clustering of proteins associated with different junctional complexes suggests that seemingly unrelated proteins might interact at cell-cell adhesions to regulate intercellular force transmission and monolayer dynamics.

\section{Cell monolayers explore distinct mechanical phenotypes}

The unsupervised clustering analysis of physical properties (Figure 3B) and siRNA perturbations (Figure 3C) can be used to re-organize the original data matrix (Figure 3A) into distinct mechanical phenotypes (Figure 3D). The result is a $q \times n$ matrix in which siRNAs (rows) and physical properties (columns) are ordered according to the results from the cross-correlation and unsupervised clustering algorithms. This analysis identified three distinct mechanical phenotypes. The most common phenotype, which we call high-speed/low-force phenotype, was characterized by an increase in physical properties associated with kinematics and a decrease in physical properties associated with forces. This phenotype was expressed when proteins from adherens junctions were knocked down. The second phenotype was also characterized by a decrease in physical properties associated with forces, but in this case kinematics remained largely unchanged. We call this phenotype low-force/same-speed. It was observed during loss of function of gap junction protein $\mathrm{Cx} 43$, tight junction proteins Cldn8, ZO-3, and JAM-A, and desmosomal protein Dsc3. The third phenotype was a high-force/high-speed phenotype in which cells migrated and deformed rapidly while exerting strong cell-cell and cellsubstrate forces. This phenotype was rare, being observable only in response to siRNA against ZO-1. 


\section{The intercellular adhesome is a highly co-regulated system}

Our analysis thus far allowed us to associate specific siRNAs with specific mechanical phenotypes. This association is not necessarily mechanistic at the molecular level, however, because down-regulation of one specific protein may lead to changes in physical properties indirectly through the action of other co-regulated proteins. To address co-regulation we selected a subset of siRNA perturbations from our library and measured the effect of these perturbations on the concentrations of cell-cell adhesion proteins. We restricted our attention to siRNAs targeting cadherins and cytoplasmic proteins. For each of these siRNA perturbations, we measured protein concentrations relative to control experiments for E-, N-, P-cadherin, $\beta$-catenin, $\alpha$-catenin, p120, ZO-1, and occludin.

Measurements of protein concentrations showed that each targeted protein was effectively down-regulated with an average knock-down efficiency of $86.3 \% \pm 14.5 \%$ (mean+SEM) (Figure S4B). As expected in a highly co-regulated system (Kumper and Ridley, 2010; Ribeiro et al, 2013), each siRNA altered not only the concentrations of the targeted proteins but also the concentrations of non-targeted proteins. For example, siRNAs against p120 caused a substantial decrease in the concentrations of all cadherins and catenins. To study co-regulation patterns systematically we followed the same procedure described for physical properties; we computed the correlation between pairs of proteins by calculating the cosine similarity between the vectors of $\mathrm{z}$-scores of protein concentrations (columns in Figure $4 \mathrm{~A}$ ). We then ordered the resulting $k \times k$ correlation matrix ( $k=8$ proteins) using the unsupervised clustering algorithm (Figure 4B). All proteins associated with adherens junctions, with the exception of $\mathrm{N}$-cadherin, showed correlations that were highly variable but exclusively positive. These findings show that cadherins and catenins considered in this study are generally not involved in compensatory feedback loops, in which a decrease in expression of one protein would be systematically compensated by an increase in expression of another one. Instead, expression levels of different adherens junction proteins in response to siRNA perturbations varied in parallel. N-cadherin and occludin were the exception to this rule and showed expression patterns that were either uncorrelated or anticorrelated with those of other adherens junction proteins.

\section{P-cadherin is predictive of the magnitude of intercellular tension, while E-cadherin is predictive of its buildup rate}

We next asked whether protein concentrations might be predictive of the physical properties that characterize epithelial dynamics. As the simplest possible predictive model we considered a linear relationship between any physical property $X_{j}$ and any protein concentration $\left[p_{i}\right]$ :

$$
X_{j}=A+B\left[p_{i}\right] \quad \text { Eq. } 1
$$

where $A$ and $B$ are constant coefficients for each pair of protein/physical property. 
To assess the predictive power of this model we performed a leave-one-out crossvalidation (LOOCV) analysis (Experimental Procedures). LOOCV analysis revealed that simple linear models involving only one protein were generally poor predictors of physical properties. None of the protein concentrations was able to predict kinematic properties such as monolayer expansion, cellular velocity, or deformation rates. Protein concentrations were also unable to predict the magnitude of traction forces. By contrast, concentrations of E- and P-cadherin were significant predictors of intercellular tension and its time evolution. P-cadherin -but not E-cadherin- was a significant predictor of the average intercellular tension $\sigma_{x x}^{m}$ (Figure 4C Table S2) as well as of intercellular tension at the end of the experiment $\sigma_{x x}^{f}$ (Figure 4D, Table S2). By contrast, E-cadherin -but not P-cadherin- was a significant predictor of the rate at which intercellular tension built up (Figure 4E, Table S2). Surprisingly, these findings suggest that P-cadherin and Ecadherin play fundamentally distinct roles in controlling force transmission at intercellular junctions. Specifically, they suggest that P-cadherin is involved in the control of equilibrium values of intercellular tension, while E-cadherin is involved in the control of the rate at which intercellular tension varies over time.

\section{P-cadherin and E-cadherin show distinct responses to mechanical stress}

How do two proteins from the same family play such fundamentally different roles in the regulation of intercellular tension? An appealing possibility is that mechanical tension triggers distinct feedback loops depending on whether it is applied through E-cadherin or $\mathrm{P}$-cadherin. To test this possibility we coated magnetic beads with either P- or E-cadherin and allowed them to bind to cadherin receptors at the apical surface of an MCF10A monolayer. We then used magnetic tweezers to subject the cell-bead junction to a pattern of oscillatory pulling forces (Figure 5A) (Bausch et al., 1999; le Duc et al., 2010; Tabdili et al., 2012). By tracking bead trajectories during force application we studied the mechanical response of the cell to forces exerted through E- or P-cadherin receptors. Pulling on beads coated with E-cadherin led to a pattern of oscillatory bead displacements whose amplitude decreased with time (Figure 5B,C). This type of cellular response to force has been extensively studied using beads coated with either extracellular matrix proteins (Riveline et al., 2001; Roca-Cusachs et al., 2009) or cadherins (le Duc et al., 2010; Tabdili et al., 2012); it indicates that tension triggers internal feedback loops that allow the cell to actively adapt to the extracellular force by reinforcing its structural connection with the bead. By contrast, pulling on beads coated with P-cadherin led to a pattern of oscillatory displacements of constant amplitude (Figure 5B,C), thus indicating that P-cadherin junctions do not reinforce. These experiments show that $\mathrm{P}$-cadherin and $\mathrm{E}$-cadherin play distinct control roles by activating distinct feedback loops downstream of intercellular tension.

\section{A linear combination of cadherin and catenin concentrations is a significant predictor of intercellular forces.}

Our previous analysis showed that the concentration of E- or P-cadherin alone is sufficient to predict significantly intercellular tension and its time evolution. We next asked whether a combination of protein concentrations is a better predictor of monolayer 
mechanics than a single protein concentration. As the simplest model involving multiple proteins, we assumed that any given physical property $X_{j}$ can be predicted by a linear combination of the concentrations of $N$ proteins $(N=2-8)$ :

$$
X_{j}=A+\sum_{i=1}^{N} B_{i}\left[p_{i}\right] \quad \text { Eq. } 2
$$

To test the predictive power of each combination, we conducted again a LOOCV analysis. We note that a cross-validation analysis is conceptually distinct from a simple linear fit; while adding more parameters to a model always implies a better fit, it does not necessarily imply higher predictive power (in fact, it often leads to lower predictive power due to overfitting).

Similarly to the case of one-protein models, N-protein models were largely unable to predict cell velocities, cell deformations, and cell tractions. By contrast, several linear combinations of multiple protein concentrations were predictive of average intercellular tension, intercellular tension at the end of the experiment, and intercellular tension buildup rate (Table S3). These linear combinations, which led to smaller prediction errors than one-protein models, always included at least one cadherin, but sometimes the three of them. In addition, they generally involved $\beta$-catenin, $\alpha$-catenin, or $\mathrm{p} 120$. The large number of N-protein models that were found to predict intercellular tension and its buildup rate highlights the fact that proteins comprising adherens junctions are coregulated. Neither ZO-1 nor occludin appeared in any of the significant models of intercellular tension magnitude or rate, indicating that these proteins are not involved in the control of these physical properties.

\section{Discussion}

Epithelial dynamics is traditionally interpreted in the context of a smooth or abrupt transition between epithelial and mesenchymal states; as the concentrations of intercellular adhesion proteins decrease, cells within tissues lose their cohesiveness and speed up their migration velocity (Cano et al., 2000; Thiery et al., 2009). The generality of this interpretation remains controversial (Montell, 2008; Revenu and Gilmour, 2009), however, and the underlying physical forces have eluded systematic experimental observation. Here we studied monolayer dynamics in response to a minimal library of siRNAs targeting the main molecular players of the intercellular adhesome. Based on the measurement of a variety of physical properties, we established that the monolayer accesses a rich diversity of dynamic phenotypes.

As predicted by the classical view of epithelial tissue dynamics, loss of function of adherens junction proteins, including cadherins, catenins, and vinculin, led to increased migration speed and increased deformation rates. These kinematic changes were paralleled by a decrease in monolayer tension. This phenotype was far from universal, however. Indeed, a variety of siRNAs targeting mostly gap junctions, tight junctions and desmosomes, led to decreased forces but virtually no changes in monolayer kinematics. Moreover, we identified a rare but highly significant phenotype in which both forces and kinematics increased. The identification of these three phenotypes establishes that the 
phenotypic plasticity of monolayer dynamics is much richer than previously thought; by expressing distinct combinations of cell-cell adhesion proteins, cells are able to control their velocity and intercellular tension independently. From a purely physical perspective, this finding is striking; any form of matter, living or inert, is expected to exhibit a welldefined relationship between forces and velocities. In the context of tissue dynamics, the existence of such universal relationship continues to evade experimental and theoretical investigation.

Cell-cell adhesion proteins display some of the highest levels of connectivity among the human proteome. For example, E-cadherin has 96 known binding partners and $\beta$-catenin has 242 (Stark et al., 2006). In this context, it is clear that predicting tissue dynamics from knowledge of molecular-scale architecture is a virtually impossible task. By contrast, here we showed that simple linear models based on the bulk measurement of protein concentrations are reliable predictors of intercellular tension and its buildup rate. Strikingly, models based on one single protein were sufficient to yield significant predictions, but models based on a larger number of proteins were more precise. These models can become a useful tool to predict tissue mechanics in physiological contexts in which intercellular tension -but not protein concentrations- is experimentally inaccessible. In contrast with the case of intercellular tension, our models were unable to predict kinematic properties such as cell velocities, deformation rates, and monolayer spreading. This absence of predictive power shows that these physical properties are controlled by protein modules that are generally not co-regulated with the intercellular adhesome.

The relationship between the magnitude of intercellular forces and the concentrations of intercellular proteins has been the subject of much controversy over the past decade (Foty and Steinberg, 2005; Liu et al., 2010; Maruthamuthu et al., 2011; Tseng et al., 2012; Maitre et al., 2012). Even experiments based on nearly identical approaches have led to conflicting conclusions as regards the existence of a correlation between intercellular force and the localization of E-cadherin at cell-cell junctions (Liu et al., 2010; Maruthamuthu et al., 2011). Our findings suggest that this discrepancy might be explained by different confounding factors. First, E-cadherin should not be regarded as the only cadherin involved in the regulation of intercellular tension; other cadherins, such as P-cadherin, should be taken into account to understand force balance at intercellular junctions. Moreover, our findings establish that different cadherins play distinct mechanical roles and trigger different feedback loops to control intercellular force transmission. Finally, our findings show that the total protein concentration, and not necessarily the amount of protein that localizes at intercellular junctions, is a robust determinant of the magnitude of intercellular tension. Once these considerations are taken into account, our study shows that simple models can predict monolayer tension with high significance.

We identified distinct roles for E-cadherin and P-cadherin as regards their ability to predict tissue dynamics. Specifically, the concentration of P-cadherin was a significant predictor of the magnitude of tension in the monolayer whereas the concentration of E- 
cadherin was a significant predictor of the rate of tension buildup. In simple terms, these predictions indicate that P-cadherin governs "how much" tension the monolayer holds while E-cadherin governs "how fast" tension grows. By using bead pulling experiments, we showed that the distinct behavior of P- and E-cadherin can be mechanistically explained by the fact that forces applied to E-cadherin trigger reinforcement feedback loops, while forces applied to P-cadherin do not. Consequently, a higher concentration of E-cadherin accelerates reinforcement responses, and thus allows faster buildup of intercellular tension. By contrast, a higher concentration of P-cadherin simply allows for a higher intercellular tension at equilibrium.

The simultaneous expression of two distinct proteins, one proportional to the magnitude of a physical quantity and one proportional to the time derivative of this quantity, provides the cell with advanced control capabilities. In proportional control systems, which are the simplest control systems involving feedback loops, the control signal is proportional to the difference between the value of the output and its desired setpoint (Bechhoefer, 2005). Control theory shows that the efficiency of this control strategy is significantly improved if the control signal also takes into account the time derivative of the output, or in other words, how fast the desired setpoint is being reached (Bechhoefer, 2005; Cloutier and Wellstead, 2010). Derivative control action of this kind is widely used in engineering to compensate rapid changes in the output and to reduce instabilities. Here we showed that the concentrations of $\mathrm{P}$ - and E-cadherin are good predictors of intercellular tension and its time derivative. Our findings thus demonstrate that the elementary building blocks required for advanced control strategies involving the combination of proportional and derivative terms are readily available to the cell.

\section{Experimental Procedures}

\section{MCF10A cell culture}

MCF10A cells were grown on DMEM-F12 media supplemented with 5\% Horse Serum, $100 \mathrm{U} / \mathrm{mL}$ Penicillin, $100 \mu \mathrm{g} / \mathrm{mL}$ Streptomycin, 20ng/mL EGF, $0.5 \mathrm{mg} / \mathrm{mL}$ Hydrocortisone, $100 \mathrm{ng} / \mathrm{mL}$ Cholera Toxin, and $10 \mathrm{ug} / \mathrm{mL}$ Insulin.

\section{Polyacrylamide gel substrates}

Polyacrylamide gels with a Young's modulus of $12 \mathrm{kPa}$ were prepared as described previously (Kandow et al., 2007; Serra-Picamal et al., 2012; Yeung et al., 2005). Briefly, a solution containing $19 \%$ acrylamide, $8 \%$ bis-acrylamide, $0.5 \%$ ammonium persulfate, $0.05 \%$ tetramethylethylenediamine, $0.64 \%$ of $200-\mathrm{nm}$-diameter red fluorescent carboxylate-modified beads and $2 \mathrm{mg} / \mathrm{mL} \mathrm{NH}$-acrylate was prepared and allowed to polymerize. After polymerization, gels were incubated with $0.1 \mathrm{mg} / \mathrm{mL}$ of collagen I overnight.

\section{PDMS membranes}

Polydimethylsiloxane (PDMS) membranes were fabricated according to procedures described previously (Ostuni et al., 2000; Poujade et al., 2007; Serra-Picamal et al., 2012). Briefly, SU8-50 masters containing rectangles of $300 \times 2,500 \mu \mathrm{m}$ were raised using conventional photolithography. Uncured PDMS was spin-coated on the masters to a 
thickness lower than the height of the SU8 feature $(35 \mu \mathrm{m})$ and cured for $2 \mathrm{hr}$ at $60{ }^{\circ} \mathrm{C}$. A thick border of PDMS was left at the edges of the membranes for handling purposes. PDMS was then peeled off from the master and kept in ethanol at $4^{\circ} \mathrm{C}$ until use.

\section{Monolayer patterning}

To pattern the cells on top of the polyacrylamide gels, a PDMS membrane was deposited on top of the polyacrylamide gel and 20,000 cells were seeded within the rectangle defined by the PDMS stencil. Cells were allowed to adhere and proliferate on the gel until confluence. 40 minutes before time lapse analysis, the PDMS membrane was carefully removed allowing the cells to migrate toward the freely available substrate.

\section{siRNAs transfection}

siRNA reverse transfections were performed by mixing Lipofectamine RNAiMAX Reagent with 100 pmoles of a pool of 3 siRNAs and 450,000 freshly trypsinized MCF10A cells (see Table S4 for siRNA sequences). Cells were then seeded on 6-well plates. 5 days after transfection cells were trypsinized and seeded on soft polyacrylamide gels. $\sim 90 \%$ of the cells were successfully transfected as measured using Block-it Alexa Fluor Red Fluorescent Oligo.

\section{RT-PCR}

RT-PCR experiments were performed according to the manufacturer's instructions (Applied Biosystems). Total mRNA was extracted from MCF10A control and knockdown cells using the PARIS kit. RNA samples were reverse-transcribed into cDNA using the High Capacity RNA-to-cDNA master mix. Taqman Gene Expression Assays were used to detect endogeneous mRNA levels. The housekeeping gene rps 18 was used as an endogeneous control for normalization (see Table 4 for Taqman probe list). PCR was performed on a 7500 Fast Real Time PCR System (Applied Biosystems).

\section{Protein quantification}

Protein expression levels were measured using Western Blot. Cells were lysed for protein extraction using lysis buffer (Tris 20mM pH8, NaCl $150 \mathrm{mM}$, EDTA 1mM, EGTA $1 \mathrm{mM}, 1 \%$ Triton-X100, antipaine $1 \mu \mathrm{g} / \mathrm{mL}$, pepstatine $1 \mu \mathrm{g} / \mathrm{mL}$, benzamidine $15 \mu \mathrm{g} / \mathrm{mL}$, leupeptine $1 \mu \mathrm{g} / \mathrm{mL}$, orthovanadate $0.1 \mathrm{mM}$ ). Cell lysates were then mixed with Laemli $1 \mathrm{X}$ and heated at $95^{\circ} \mathrm{C}$ for 5 minutes. Next, cell lysates were loaded to $4-20 \%$ polyacrylamide gels (Bio-Rad) for electrophoresis. Proteins were then transferred to a nitrocellulose membrane (Whatman, GE Healthcare Life Sciences), which was blocked with 5\% drymilk-Tris Buffer saline- $0.2 \%$ Tween, and incubated with primary antibodies (overnight at $4^{\circ} \mathrm{C}$ ) followed by the horseradish peroxidase coupled secondary antibodies ( $1 \mathrm{~h}$, room temperature). Bands were revealed using the LumiLight kit (Roche) and the intensity of the bands was quantified using ImageJ software. Tubulin was used as an endogeneous control for normalization. Protein concentrations are reported relative to the control.

\section{Immunostaining}

MCF10A cells were washed with PBS, fixed with 3\% paraformaldehyde for 10 minutes and permeabilized in $0.1 \%$ triton X-100 for 5 minutes. Cells were blocked in $10 \%$ FBS for 1 hour before being incubated for 4 hours with primary antibodies. After incubation 
with the appropriate fluorescence-conjugated secondary antibodies, cells were washed and mounted in Mowiol reagent. Images were acquired with a Nikon C1Si confocal microscope, using a $60 \times 1.4 \mathrm{NA}$ lens.

\section{Antibodies}

The primary antibodies used were: anti-Ecadherin monoclonal antibody (clone 36, BD Transduction Laboratories), anti-Ncadherin monoclonal antibody (clone $8 \mathrm{C} 11$, Thermo Scientific), anti-Pcadherin monoclonal antibody (clone 6A9, Upstate), anti- $\beta$ catenin monoclonal antibody (clone 14, BD Transduction Laboratories), anti-acatenin monoclonal antibody (clone 15D9, Enzo Life Sciences), anti-p120 catenin monoclonal antibody (clone 98, BD Transduction Laboratories), anti-Occludin monoclonal antibody (clone OC-3F10, Invitrogen), anti-ZO1 rabbit polyclonal antibody (Invitrogen), antiaTubulin monoclonal antibody (clone B-5-1-2, Sigma-Aldrich). The secondary antibodies used for western blots were: peroxidase-conjugated anti-mouse $\operatorname{IgG}$ and peroxidase-conjugated anti-rabbit IgG (Jackson Immuno Research). The secondary antibodies used for immunofluorescence were: Alexa Fluor 488 anti-rabbit (Invitrogen, molecular probes) and Alexa Fluor 488 anti-mouse (Invitrogen, molecular probes). Factin was stained with phalloidin-TRITC (Sigma-Aldrich).

\section{Time-lapse microscopy}

Multidimensional acquisitions were performed on an automated inverted microscope (Nikon Eclipse Ti, 10× lens) equipped with thermal, $\mathrm{CO}_{2}$, and humidity control, using MetaMorph (Universal Imaging) software. Images were obtained every 3 minutes during 450 minutes. Up to 15 independent monolayers were imaged in parallel using a motorized XY stage.

\section{Particle Imaging Velocimetry and strain rate calculation}

Monolayer velocity fields were computed using a custom-made particle imaging velocimetry software. To reduce systematic biases in subpixel resolution and peaklocking effects, we implemented an iterative process (up to 4 iterations) based on a continuous window shift technique (Serra-Picamal et al., 2012).

Strain-rate was $\varepsilon_{x x}$ was calculated using the following expression (Blanchard et al., 2009; Serra-Picamal et al., 2012):

$$
\dot{\varepsilon_{x x}}=\frac{d V_{x}}{d x} \quad \text { Eq. } 3
$$

\section{Traction Force microscopy}

Traction forces were computed using Fourier Transform Traction Microscopy with finite gel thickness (Trepat et al., 2009). Gel displacements between any experimental time point and a reference image obtained after cell trypsinization were computed using particle imaging velocimetry software described above.

\section{Monolayer Stress microscopy}


Monolayer Stress Microscopy is based on the principle that, according to Newton's laws, traction forces applied at the cell-gel interface must be balanced by intra- and intercellular forces (Serra-Picamal et al., 2012; Tambe et al., 2013; Tambe et al., 2011). In a $2 \mathrm{D}$ approximation, monolayer stress is fully captured by a tensor possessing two independent normal components $\left(\sigma_{x x}\right.$ and $\left.\sigma_{y y}\right)$ and two identical shear components $\left(\sigma_{x y}\right.$ and $\left.\sigma_{y x}\right)$. At every pixel of the monolayer, these four components of the stress tensor define two particular directions of the plane, one in which the stress is maximum and one in which it is minimum. These directions, which are mutually orthogonal, are called principal stress orientations, and the stress values in each principal orientation are called maximum $\left(\sigma_{11}\right)$ and minimum $\left(\sigma_{22}\right)$ stress components. The average normal stress is defined as $\sigma_{n}=\left(\sigma_{11}+\sigma_{22}\right) / 2$, while the maximum shear stress is defined as $\sigma_{s}=\left(\sigma_{11}-\sigma_{22}\right) / 2$.

\section{Magnetic tweezers}

Magnetic tweezers experiments were carried out as previously described (Roca-Cusachs et al., 2013; Roca-Cusachs et al., 2009). Briefly, $3 \mu \mathrm{m}$ diameter magnetic beads precoated with protein $\mathrm{G}$ (Novex) were covalently coated with purified E-cadherin-Fc or Pcadherin-Fc proteins. The beads were first washed with NaPhosphate buffer $(0.1 \mathrm{M}, \mathrm{pH}$ 8 ), incubated with $20 \mu \mathrm{g}$ of the $\mathrm{Fc}$-Tagged proteins for $2 \mathrm{hr}$, and then with crosslinking buffer for $1 \mathrm{hr}(25 \mathrm{mM}$ DMP, $0.2 \mathrm{M}$ triethanolamine, $\mathrm{pH} 8.2)$. The protein-coated beads were allowed to settle on a confluent monolayer of MCF10A cells for 30 minutes before starting the experiment. To measure the extent of reinforcement, a $0.2 \mathrm{nN}$ pulsatory force was applied to beads attached to cells. Bead movement in response to the pulsatory force was tracked using a custom-made tracking software. Stiffness of the cell-bead contact was calculated as the ratio between the magnitude of the applied force and that of the observed bead oscillation.

\section{Averaging}

Physical properties of cells and cell monolayers often exhibit broad non-Gaussian distributions with log-normal or exponential tails (Fabry et al., 2003; Li et al., 2008; Trepat et al., 2009). To avoid potential averaging artifacts caused by extreme data in these tails, we first computed the median -rather than the mean- of any physical property across space. We then averaged medians over experimental repeats and/or time. In the case of cell velocities, which average to roughly zero due to the symmetry of monolayer expansion, we computed the median of absolute values.

\section{Computation of z-scores}

The $\mathrm{z}$-score (or standard score in statistics) is defined as the signed number of standard deviations an observed quantity deviates from the mean of that quantity (Birmingham et al., 2009). In the context of this study, the z-score of a quantity $x$ (a physical property in Figure $3 \mathrm{~A}$ or a protein concentration in Figure 4A) in response to a siRNA perturbation is defined as:

$$
Z=\frac{\bar{x}-\overline{x_{c}}}{\sigma_{c}} \quad \text { Eq. } 4
$$

where $\bar{x}$ is the mean of $x$ under the siRNA perturbation, $\overline{x_{c}}$ is the mean of $x$ under control conditions, and $\sigma_{c}$ is the standard deviation of $x$ under control conditions. 


\section{Computation of correlation matrices}

As a measure of correlation we used cosine similarity between pairs of vectors containing $z$-scores of physical properties. Consider a matrix $Z$ containing the $z$-scores of $n$ physical properties under $m$ siRNA perturbations ( $m$ rows and $n$ columns). Each column of the matrix defines a m-dimensional vector $\vec{P}$ that contains the z-scores of one physical property. To assess the correlation between two physical properties $i$ and $j$ we computed the cosine of the angle $\theta_{i j}$ between the vectors $\vec{P}_{l}$ and $\vec{P}_{j}$ (vectors that contain the z-scores of properties $i$ and $j$ ):

$$
\cos \left(\theta_{i j}\right)=\frac{\overrightarrow{P_{l}} \cdot \overrightarrow{P_{j}}}{\left\|P_{i}\right\|\left\|P_{j}\right\|} \quad \text { Eq. } 5
$$

When repeated over each possible pair of physical properties, this operation yields a $n \times n$ correlation matrix $C$ in which each element is defined as $C_{i j}=\cos \left(\theta_{i j}\right)$. This matrix is shown is Figure 3B. In a similar way, we also computed a $q \times q$ correlation matrix of siRNA perturbations (Figure $3 \mathrm{C}$ ) and a $k \times k$ correlation matrix of protein concentrations (Figure 4B).

\section{Unsupervised clustering analysis}

Unsupervised clustering analysis was performed as described in Sales-Pardo et al (SalesPardo et al., 2007). Briefly, we first ordered the correlation matrix to place high correlation values close to the diagonal and low correlation values close to the matrix edges. Second, we obtained clusters by identifying diagonal blocks containing elements of high correlation. To this end, we assumed a matrix model that is block diagonal in which matrix elements in each block are equal and off-diagonal elements are also equal. We then obtained the block diagonal model that fits best our ordered matrix according to the Bayesian Information Criterion.

\section{Leave-one-out cross-validation}

To assess the predictive power of each model we performed a LOOCV analysis (Simon, 2007). In this analysis, the value of a physical property under a given siRNA condition is predicted using a linear combination of the protein concentrations in that condition and the parameters $A$ and $B_{i}$ (see Eqs. 1 and 2). These parameters are obtained by fitting a linear model to all siRNA conditions except for the one to be predicted. This procedure is repeated for every siRNA condition and the total prediction error is then computed as the mean squared error of all predictions. To establish significance of the predictions and ensure that low prediction errors are not merely an outcome of multiple-testing, we run randomization tests on the values of the physical properties and keep only models whose prediction error is significantly low at a 5\% level. This approach is typically used to avoid overfitting when the number of potential explanatory variables (in our case protein concentrations) is of the order of the number of conditions (in our case siRNAs) under which predictions are sought.

\section{Acknowledgements}


We thank F. Supek, B. Lehner, A. Brugués and R. Vincent for discussions, R. Zaidel-Bar for sharing unpublished data, and E. Sahai for sharing reagents. This research was supported by the Spanish Ministry for Science and Innovation (BFU2012-38146, XT), the European Research Council (Grant Agreement 242993, XT), and the National Institutes of Health (R01HL107561, XT).

\section{Author contributions}

E.B and X.T conceived the study and designed experiments. E.B., M.B., and A.E performed experiments. E.B, V.C, and A.E analyzed data. X.S and P.R developed data analysis tools. V.C. and J.J.M. developed computational mechanics tools. M.S and R.G performed unsupervised clustering analysis and LOOCV analysis. E.B., M.S, R.G, and X.T. wrote the manuscript. All authors discussed and interpreted results and commented on the manuscript.

\section{Figure Captions}

Figure 1. As the monolayer increases its cohesiveness, cell migration slows down and physical forces buildup. Localization of ZO-1, E-cadherin, P-cadherin, $\alpha$-catenin, and Factin at the leading edge $(A, B)$ and center $(C, D)$ of the monolayer at $\mathrm{t}=0 \mathrm{hr}(\mathrm{A}, \mathrm{C})$ and $\mathrm{t}=8 \mathrm{hr}(\mathrm{B}, \mathrm{D})$. Phase contrast images $(\mathrm{E}, \mathrm{F})$, maps of cell velocities $(\mathrm{G}, \mathrm{H})$, maps of traction forces $(\mathrm{I}, \mathrm{J})$, and maps of monolayer tension $(\mathrm{K}, \mathrm{L})$ at $\mathrm{t}=0 \mathrm{hr}(\mathrm{E}, \mathrm{G}, \mathrm{I}, \mathrm{K})$ and $\mathrm{t}=8 \mathrm{hr}(\mathrm{F}, \mathrm{H}, \mathrm{J}, \mathrm{L})$. Time evolution of monolayer area $(\mathrm{M})$, cell velocity $(\mathrm{N})$, strain energy density $(\mathrm{O})$, and median monolayer tension $(\mathrm{P})$. Scale bar $=20 \mu \mathrm{m}(\mathrm{A}-\mathrm{D})$, scale bar $=100 \mu \mathrm{m}(\mathrm{E}-\mathrm{L})$. Data are presented as mean $\pm \operatorname{SEM}(\mathrm{n}=13)$.

Figure 2. Downregulation of cell-cell adhesion proteins alter monolayer dynamics. Representative maps showing the effect of siRNAs on monolayer dynamics after $8 \mathrm{hr}$ of expansion. For each siRNA, each row displays contrast images (first row), monolayer velocity (second row), traction force (third row), and intercellular tension (forth row). Panels show the control case $(\mathrm{A}, \mathrm{G})$ and selected siRNAs targeting adherens junctions (BF), tight junctions (H-J), desmosomes (K), and gap junctions (L). Additional time points and siRNA pertubations are shown in Fig S5-S10. Time evolution of the increase in monolayer area $(M)$, average cell velocity $(N)$, strain energy density $(\mathrm{O})$, and average intercellular tension (P) for the control case and the 10 siRNAs shown in panels A-L. The time evolution of physical properties in response to all siRNAs is shown in Figure S11. Scale bar, $100 \mu \mathrm{m}$. Data are presented as mean \pm SEM $(n=3-13)$

Figure 3. Cell monolayers with perturbed cell-cell adhesions exhibit distinct mechanical phenotypes. (A) Effect of siRNAs on physical properties expressed in terms of their z-scores. (B) Correlation between physical properties computed as the cosine similarity between all possible pairs of columns in panel (A). (C) Correlation between siRNAs computed as the cosine similarity between all possible pairs of rows in panel (A). 
An unsupervised clustering algorithm was used to order rows and columns in panels (B) and (C) and to identify clusters whose separation is marked with black lines. (D) Reorganization of panel (A) into phenotypic clusters according to the unsupervised analysis of correlation matrices (B) and (C).

Figure 4. Protein concentrations predict intercellular forces and their buildup rate. (A) $\mathrm{z}$-scores of protein concentrations in response to siRNA perturbations. (B) Correlation between protein expression patterns computed as the cosine similarity between columns in panel (A). (C) The concentration of P-cadherin predicts average intercellular tension. (D) The concentration of P-cadherin predicts intercellular tension at the end of the experiment. (E) The concentration of E-cadherin predicts the rate of intercellular tension buildup. The $x$-axis in panels $\mathrm{C}$-E shows the values predicted by the 1-protein models whereas the y-axis shows the experimental values. Each data point corresponds to one siRNA perturbation. Error bars in panels are SEM. All predictions displayed in panels $\mathrm{C}$-E were significant to at least $\mathrm{p}<0.05$. See Table $\mathrm{S} 2$ for values of prediction errors. See Table S3 for predictions by N-protein models.

Figure 5. Force applied to E-cadherin triggers reinforcement feedback loops whereas force applied to P-cadherin does not. (A) Experimental setup: magnetic beads coated with E-cadherin or P-cadherin were attached to the apical surface of MCF10A monolayers and subjected to a series force pulses using magnetic tweezers. (B) Representative examples of bead displacements for P-cadherin coated beads (top) and Ecadherin coated beads (bottom). (C) Relative stiffening of the cell-bead contact for Ecadherin coated beads and P-cadherin coated beads.

\section{REFERENCES}

Abe, K., and Takeichi, M. (2008). EPLIN mediates linkage of the cadherin catenin complex to F-actin and stabilizes the circumferential actin belt. Proc Natl Acad Sci U S A 105, 13-19.

Bao, L., Sachs, F., and Dahl, G. (2004). Connexins are mechanosensitive. Am J Physiol Cell Physiol 287, C1389-1395.

Bausch, A.R., Moller, W., and Sackmann, E. (1999). Measurement of local viscoelasticity and forces in living cells by magnetic tweezers. Biophys J 76, 573-579. Bechhoefer, J. (2005). Feedback for physicists: A tutorial essay on control. Reviews of Modern Physics 77, 783-836.

Birmingham, A., Selfors, L.M., Forster, T., Wrobel, D., Kennedy, C.J., Shanks, E., Santoyo-Lopez, J., Dunican, D.J., Long, A., Kelleher, D., et al. (2009). Statistical methods for analysis of high-throughput RNA interference screens. Nature methods 6 , 569-575.

Blanchard, G.B., Kabla, A.J., Schultz, N.L., Butler, L.C., Sanson, B., Gorfinkiel, N., Mahadevan, L., and Adams, R.J. (2009). Tissue tectonics: morphogenetic strain rates, cell shape change and intercalation. Nature methods 6, 458-464.

Blanchard, G.B., Murugesu, S., Adams, R.J., Martinez-Arias, A., and Gorfinkiel, N. (2010). Cytoskeletal dynamics and supracellular organisation of cell shape fluctuations during dorsal closure. Development 137, 2743-2752. 
Borghi, N., Sorokina, M., Shcherbakova, O.G., Weis, W.I., Pruitt, B.L., Nelson, W.J., and Dunn, A.R. (2012). E-cadherin is under constitutive actomyosin-generated tension that is increased at cell-cell contacts upon externally applied stretch. Proc Natl Acad Sci U S A 109, 12568-12573.

Bosveld, F., Bonnet, I., Guirao, B., Tlili, S., Wang, Z., Petitalot, A., Marchand, R., Bardet, P.L., Marcq, P., Graner, F., et al. (2012). Mechanical control of morphogenesis by Fat/Dachsous/Four-jointed planar cell polarity pathway. Science 336, 724-727. Budnar, S., and Yap, A.S. (2013). A mechanobiological perspective on cadherins and the actin-myosin cytoskeleton. F1000Prime Rep 5, 35.

Campinho, P., Behrndt, M., Ranft, J., Risler, T., Minc, N., and Heisenberg, C.P. (2013). Tension-oriented cell divisions limit anisotropic tissue tension in epithelial spreading during zebrafish epiboly. Nat Cell Biol 15, 1405-1414.

Cano, A., Perez-Moreno, M.A., Rodrigo, I., Locascio, A., Blanco, M.J., del Barrio, M.G., Portillo, F., and Nieto, M.A. (2000). The transcription factor snail controls epithelialmesenchymal transitions by repressing E-cadherin expression. Nat Cell Biol 2, 76-83. Cloutier, M., and Wellstead, P. (2010). The control systems structures of energy metabolism. J R Soc Interface 7, 651-665.

Drees, F., Pokutta, S., Yamada, S., Nelson, W.J., and Weis, W.I. (2005). Alpha-catenin is a molecular switch that binds E-cadherin-beta-catenin and regulates actin-filament assembly. Cell 123, 903-915.

Fabry, B., Maksym, G.N., Butler, J.P., Glogauer, M., Navajas, D., Taback, N.A., Millet, E.J., and Fredberg, J.J. (2003). Time scale and other invariants of integrative mechanical behavior in living cells. Physical review 68, 041914.

Foty, R.A., and Steinberg, M.S. (2005). The differential adhesion hypothesis: a direct evaluation. Dev Biol 278, 255-263.

Friedl, P., and Gilmour, D. (2009). Collective cell migration in morphogenesis, regeneration and cancer. Nat Rev Mol Cell Biol 10, 445-457.

Harris, A.R., Peter, L., Bellis, J., Baum, B., Kabla, A.J., and Charras, G.T. (2012).

Characterizing the mechanics of cultured cell monolayers. Proc Natl Acad Sci U S A 109, 16449-16454.

Heisenberg, C.P., and Bellaiche, Y. (2013). Forces in tissue morphogenesis and patterning. Cell 153, 948-962.

Herrmann, H., Bar, H., Kreplak, L., Strelkov, S.V., and Aebi, U. (2007). Intermediate filaments: from cell architecture to nanomechanics. Nat Rev Mol Cell Biol 8, 562-573. Hur, S.S., del Alamo, J.C., Park, J.S., Li, Y.S., Nguyen, H.A., Teng, D., Wang, K.C., Flores, L., Alonso-Latorre, B., Lasheras, J.C., et al. (2012). Roles of cell confluency and fluid shear in 3-dimensional intracellular forces in endothelial cells. Proc Natl Acad Sci U S A 109, 11110-11115.

Kandow, C.E., Georges, P.C., Janmey, P.A., and Beningo, K.A. (2007). Polyacrylamide hydrogels for cell mechanics: steps toward optimization and alternative uses. Methods Cell Biol 83, 29-46.

Kumper, S., and Ridley, A.J. (2010). p120ctn and P-cadherin but not E-cadherin regulate cell motility and invasion of DU145 prostate cancer cells. PloS one 5, e1 1801.

le Duc, Q., Shi, Q., Blonk, I., Sonnenberg, A., Wang, N., Leckband, D., and de Rooij, J. (2010). Vinculin potentiates E-cadherin mechanosensing and is recruited to actin- 
anchored sites within adherens junctions in a myosin II-dependent manner. The Journal of cell biology 189, 1107-1115.

Leckband, D.E., le Duc, Q., Wang, N., and de Rooij, J. (2011). Mechanotransduction at cadherin-mediated adhesions. Curr Opin Cell Biol 23, 523-530.

Legoff, L., Rouault, H., and Lecuit, T. (2013). A global pattern of mechanical stress polarizes cell divisions and cell shape in the growing Drosophila wing disc. Development 140, 4051-4059.

Li, L., Norrelykke, S.F., and Cox, E.C. (2008). Persistent cell motion in the absence of external signals: a search strategy for eukaryotic cells. PloS one 3, e2093.

Liu, Z., Tan, J.L., Cohen, D.M., Yang, M.T., Sniadecki, N.J., Ruiz, S.A., Nelson, C.M., and Chen, C.S. (2010). Mechanical tugging force regulates the size of cell-cell junctions. Proc Natl Acad Sci U S A 107, 9944-9949.

Maitre, J.L., Berthoumieux, H., Krens, S.F., Salbreux, G., Julicher, F., Paluch, E., and Heisenberg, C.P. (2012). Adhesion functions in cell sorting by mechanically coupling the cortices of adhering cells. Science 338, 253-256.

Maitre, J.L., and Heisenberg, C.P. (2013). Three functions of cadherins in cell adhesion.

Curr Biol 23, R626-633.

Martin, A.C., Kaschube, M., and Wieschaus, E.F. (2009). Pulsed contractions of an actinmyosin network drive apical constriction. Nature 457, 495-499.

Maruthamuthu, V., Sabass, B., Schwarz, U.S., and Gardel, M.L. (2011). Cell-ECM traction force modulates endogenous tension at cell-cell contacts. Proc Natl Acad Sci U S A $108,4708-4713$.

Montell, D.J. (2008). Morphogenetic cell movements: diversity from modular mechanical properties. Science 322, 1502-1505.

Nieman, M.T., Prudoff, R.S., Johnson, K.R., and Wheelock, M.J. (1999). N-cadherin promotes motility in human breast cancer cells regardless of their E-cadherin expression. The Journal of cell biology 147, 631-644.

Ostuni, E., Kane, R.S., Chen, C.S., Ingber, D.E., and Whitesides, G.M. (2000). Patterning Mammalian Cells Using Elastomeric Membranes. Langmuir 16, 7811.

Poujade, M., Grasland-Mongrain, E., Hertzog, A., Jouanneau, J., Chavrier, P., Ladoux, B., Buguin, A., and Silberzan, P. (2007). Collective migration of an epithelial monolayer in response to a model wound. Proc Natl Acad Sci U S A 104, 15988-15993.

Rauzi, M., Lenne, P.F., and Lecuit, T. (2010). Planar polarized actomyosin contractile flows control epithelial junction remodelling. Nature 468, 1110-1114.

Revenu, C., and Gilmour, D. (2009). EMT 2.0: shaping epithelia through collective migration. Curr Opin Genet Dev 19, 338-342.

Ribeiro, A.S., Albergaria, A., Sousa, B., Correia, A.L., Bracke, M., Seruca, R., Schmitt, F.C., and Paredes, J. (2010). Extracellular cleavage and shedding of P-cadherin: a

mechanism underlying the invasive behaviour of breast cancer cells. Oncogene 29, $392-$ 402.

Riveline, D., Zamir, E., Balaban, N.Q., Schwarz, U.S., Ishizaki, T., Narumiya, S., Kam, Z., Geiger, B., and Bershadsky, A.D. (2001). Focal contacts as mechanosensors: externally applied local mechanical force induces growth of focal contacts by an mDia1dependent and ROCK-independent mechanism. The Journal of cell biology 153, 11751186. 
Roca-Cusachs, P., del Rio, A., Puklin-Faucher, E., Gauthier, N.C., Biais, N., and Sheetz, M.P. (2013). Integrin-dependent force transmission to the extracellular matrix by alphaactinin triggers adhesion maturation. Proc Natl Acad Sci U S A 110, E1361-1370.

Roca-Cusachs, P., Gauthier, N.C., Del Rio, A., and Sheetz, M.P. (2009). Clustering of alpha(5)beta(1) integrins determines adhesion strength whereas alpha(v)beta(3) and talin enable mechanotransduction. Proc Natl Acad Sci U S A 106, 16245-16250.

Runkle, E.A., and Mu, D. (2013). Tight junction proteins: from barrier to tumorigenesis. Cancer Lett 337, 41-48.

Sales-Pardo, M., Guimera, R., Moreira, A.A., and Amaral, L.A. (2007). Extracting the hierarchical organization of complex systems. Proc Natl Acad Sci U S A 104, 1522415229.

Serra-Picamal, X., Conte, V., Vincent, R., Anon, E., Tambe, D.T., Bazellieres, E., Butler, J.P., Fredberg, J.J., and Trepat, X. (2012). Mechanical waves during tissue expansion. Nature Physics 8, 628-634.

Simon, R. (2007). Resampling Strategies for Model Assessment and Selection : LeaveOne-Out-Cross-Validation. In Fundamentals of Data Mining in Genomics and

Proteomics W. Dubitzky, M. Granzow, and D.P. Berrar, eds. (Springer

Science+Business Media), pp. pp 177-178.

Simpson, K.J., Selfors, L.M., Bui, J., Reynolds, A., Leake, D., Khvorova, A., and Brugge, J.S. (2008). Identification of genes that regulate epithelial cell migration using an siRNA screening approach. Nat Cell Biol 10, 1027-1038.

Solon, J., Kaya-Copur, A., Colombelli, J., and Brunner, D. (2009). Pulsed forces timed by a ratchet-like mechanism drive directed tissue movement during dorsal closure. Cell 137, 1331-1342.

Stark, C., Breitkreutz, B.J., Reguly, T., Boucher, L., Breitkreutz, A., and Tyers, M. (2006). BioGRID: a general repository for interaction datasets. Nucleic acids research 34, D535-539.

Tabdili, H., Langer, M., Shi, Q., Poh, Y.C., Wang, N., and Leckband, D. (2012).

Cadherin-dependent mechanotransduction depends on ligand identity but not affinity. J

Cell Sci 125, 4362-4371.

Tambe, D.T., Croutelle, U., Trepat, X., Park, C.Y., Kim, J.H., Millet, E., Butler, J.P., and Fredberg, J.J. (2013). Monolayer stress microscopy: limitations, artifacts, and accuracy of recovered intercellular stresses. PloS one 8, e55172.

Tambe, D.T., Hardin, C.C., Angelini, T.E., Rajendran, K., Park, C.Y., Serra-Picamal, X., Zhou, E.H., Zaman, M.H., Butler, J.P., Weitz, D.A., et al. (2011). Collective cell guidance by cooperative intercellular forces. Nat Mater 10, 469-475.

Theveneau, E., Steventon, B., Scarpa, E., Garcia, S., Trepat, X., Streit, A., and Mayor, R. (2013). Chase-and-run between adjacent cell populations promotes directional collective migration. Nat Cell Biol 15, 763-772.

Thiery, J.P., Acloque, H., Huang, R.Y., and Nieto, M.A. (2009). Epithelial-mesenchymal transitions in development and disease. Cell 139, 871-890.

Trepat, X., and Fredberg, J.J. (2012). Plithotaxis and emergent dynamics in collective cellular migration. Trends Cell Biol 21, 638-646.

Trepat, X., Wasserman, M.R., Angelini, T.E., Millet, E., Weitz, D.A., Butler, J.P., and Fredberg, J.J. (2009). Physical forces during collective cell migration. Nature Physics 5 $426-430$. 
Tseng, Q., Duchemin-Pelletier, E., Deshiere, A., Balland, M., Guillou, H., Filhol, O., and Thery, M. (2012). Spatial organization of the extracellular matrix regulates cell-cell junction positioning. Proc Natl Acad Sci U S A 109, 1506-1511.

Wang, N., and Stamenovic, D. (2000). Contribution of intermediate filaments to cell stiffness, stiffening, and growth. Am J Physiol Cell Physiol 279, C188-194.

Weber, G.F., Bjerke, M.A., and DeSimone, D.W. (2012). A mechanoresponsive cadherin-keratin complex directs polarized protrusive behavior and collective cell migration. Dev Cell 22, 104-115.

Yamada, S., Pokutta, S., Drees, F., Weis, W.I., and Nelson, W.J. (2005). Deconstructing the cadherin-catenin-actin complex. Cell 123, 889-901.

Yeung, T., Georges, P.C., Flanagan, L.A., Marg, B., Ortiz, M., Funaki, M., Zahir, N., Ming, W., Weaver, V., and Janmey, P.A. (2005). Effects of substrate stiffness on cell morphology, cytoskeletal structure, and adhesion. Cell Motil Cytoskeleton 60, 24-34. Zaidel-Bar, R. (2013). Cadherin adhesome at a glance. J Cell Sci 126, 373-378. 


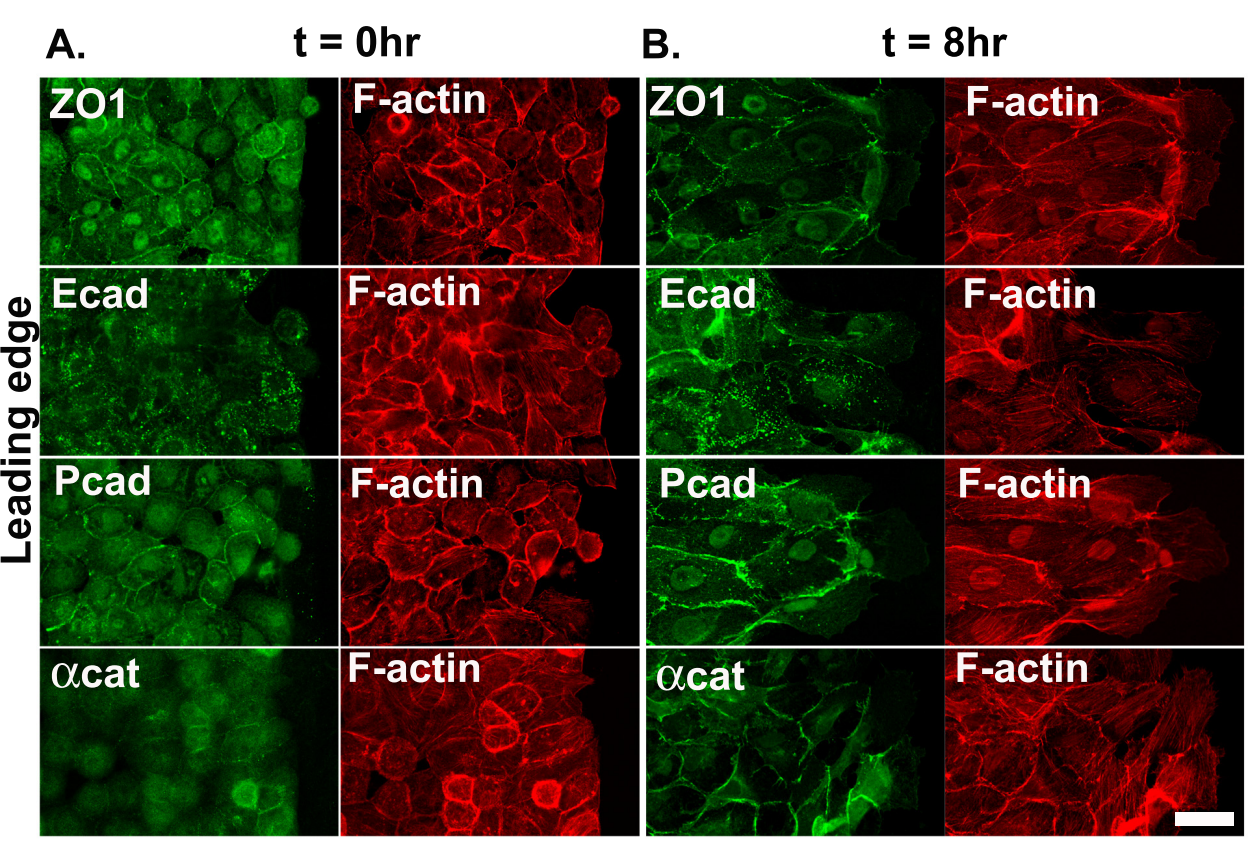

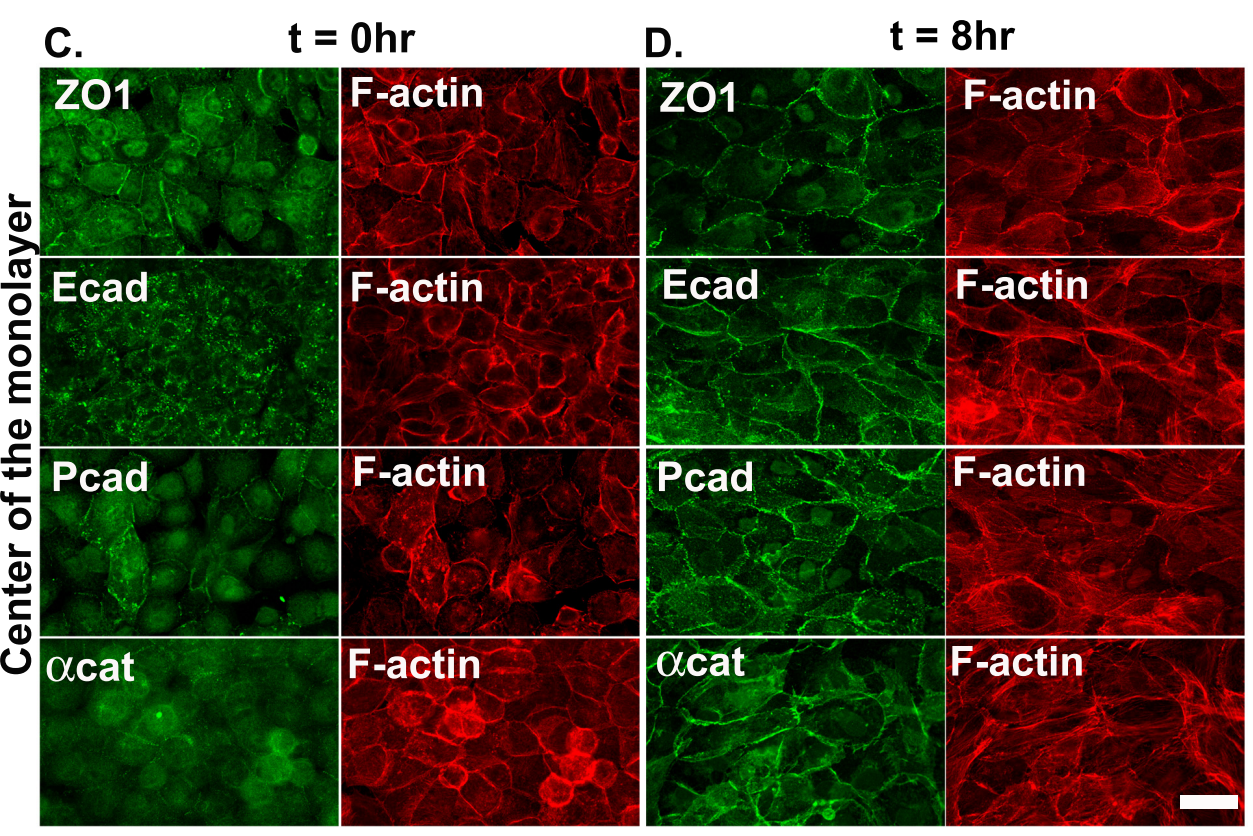
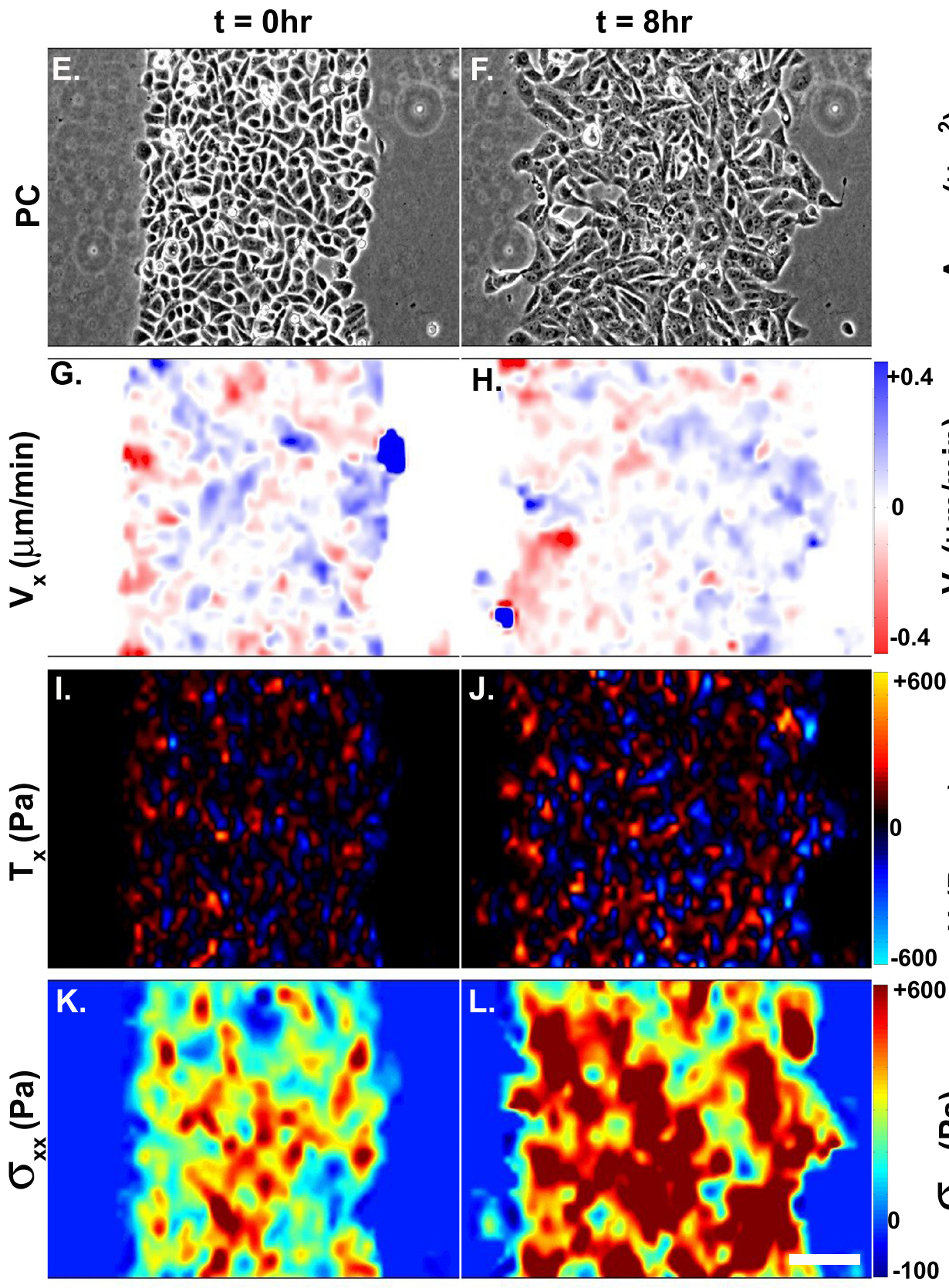

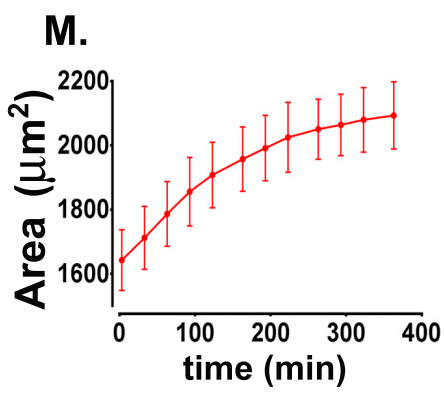

$0.4 \mathrm{~N}$.
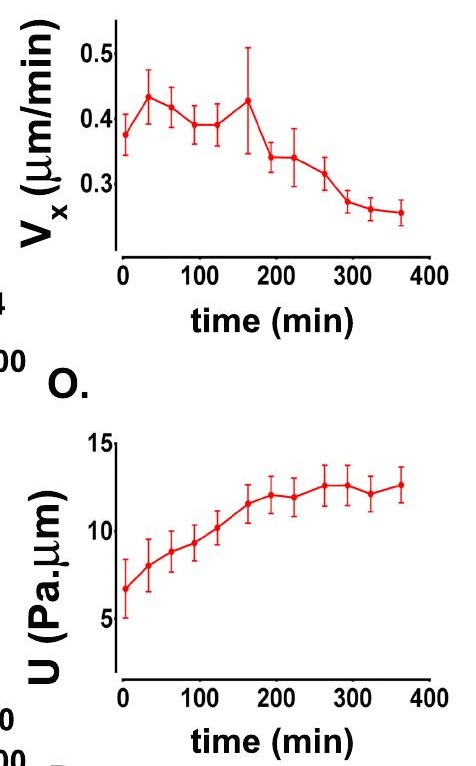
P.

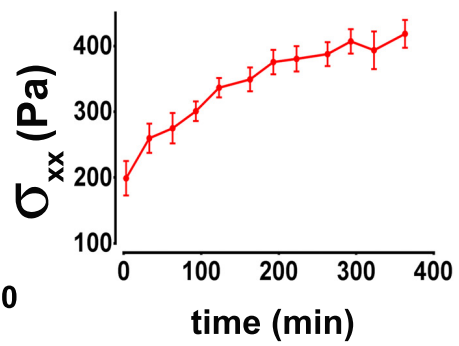

Fig 1 

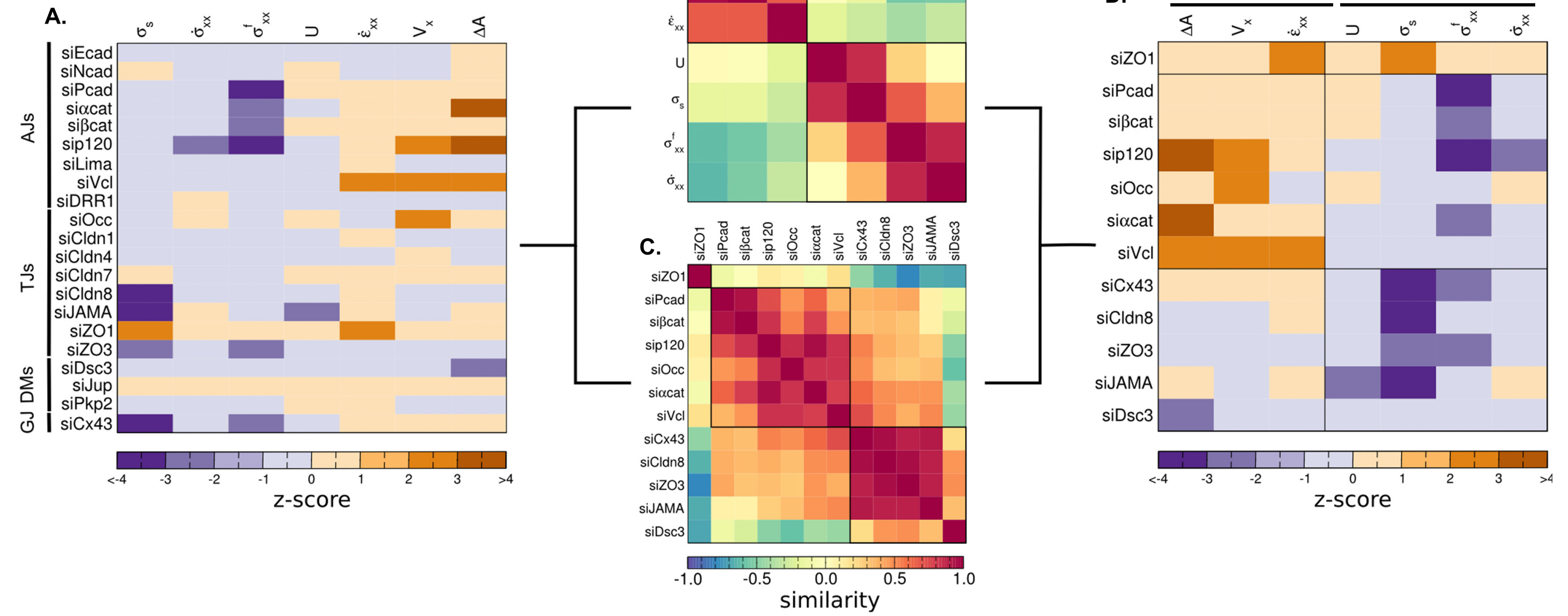

Fig 3 
Protein concentration

A.

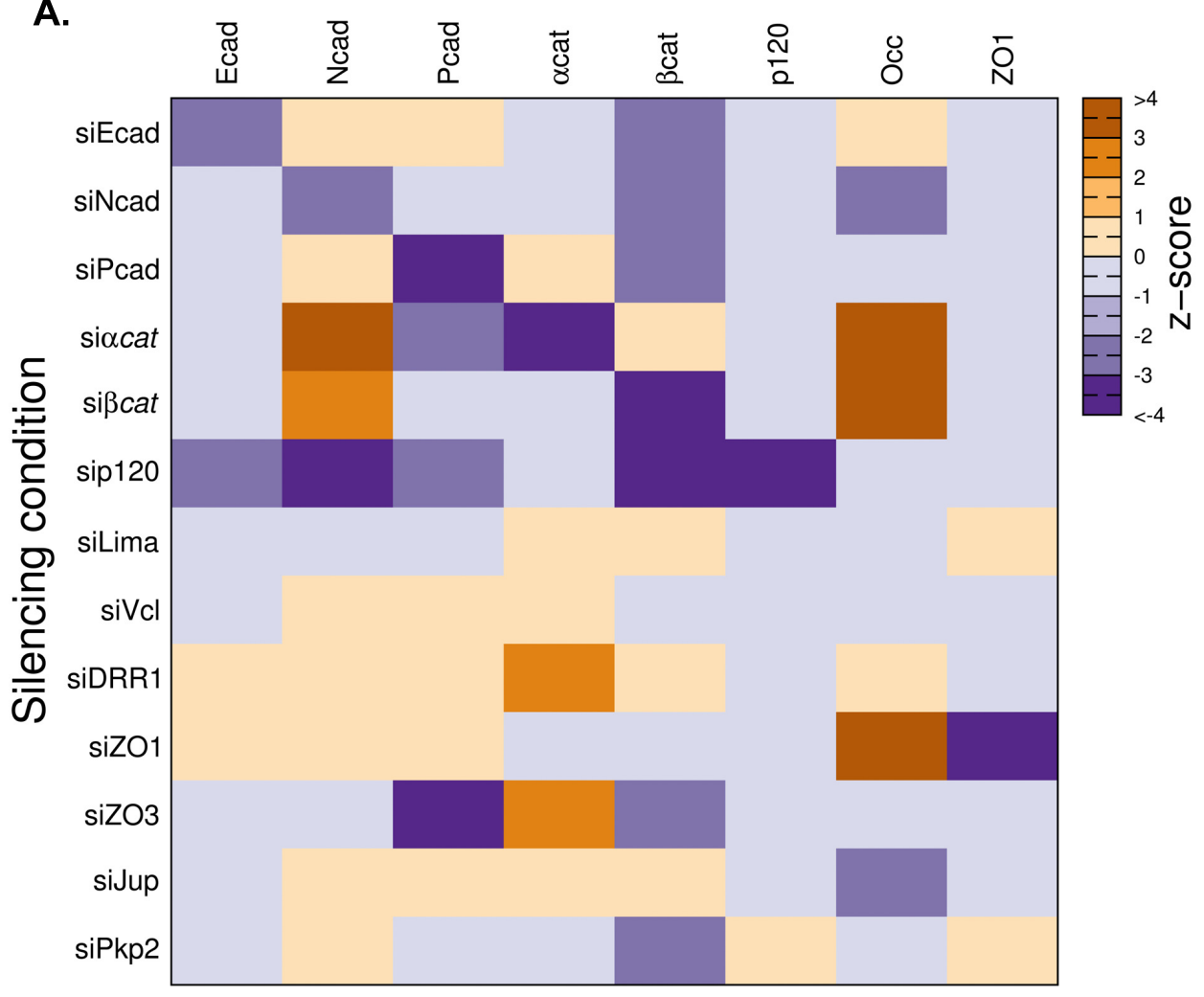

B.

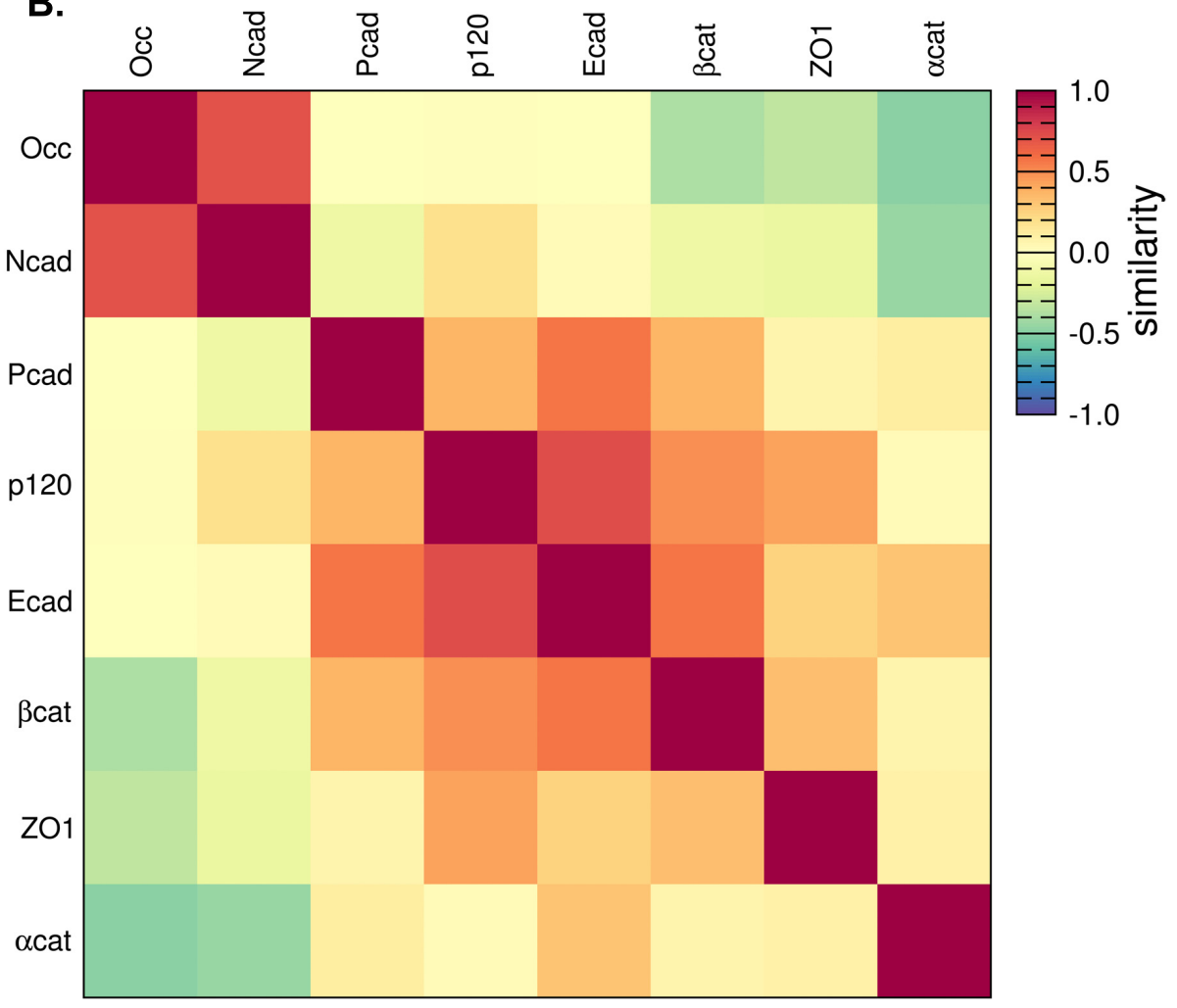

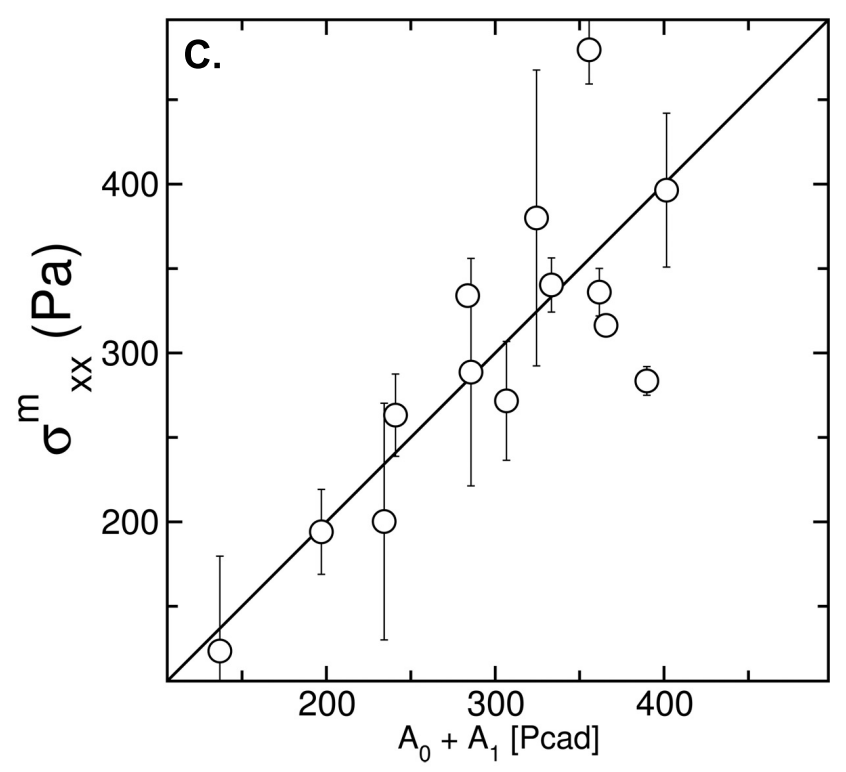
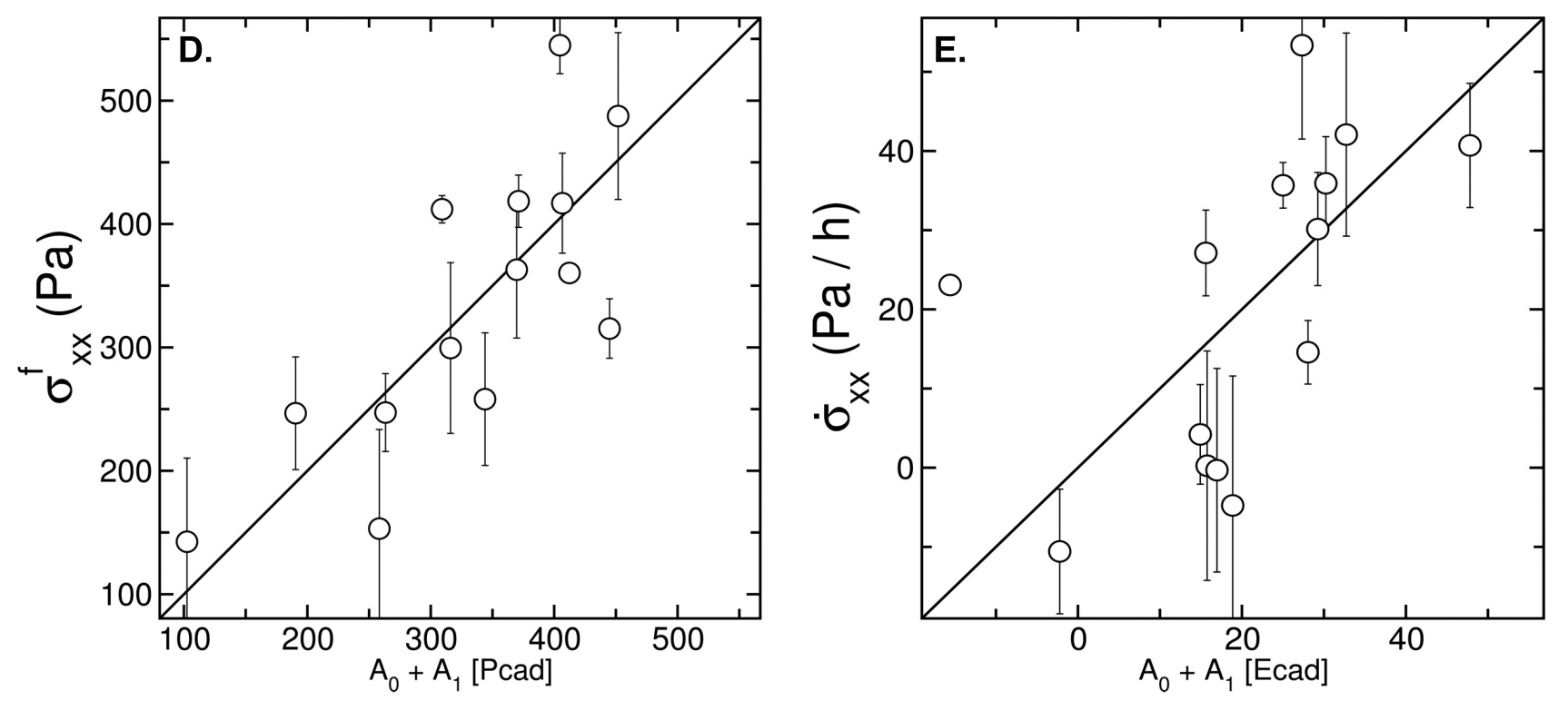

Fig 4 
A.

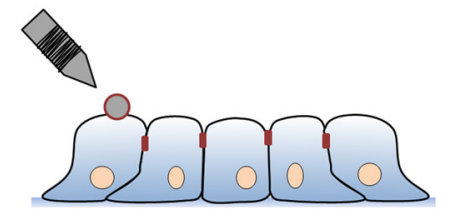

B.

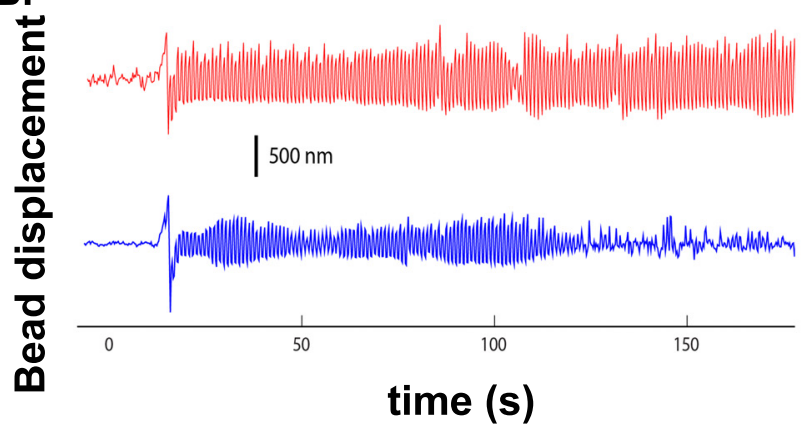

c.

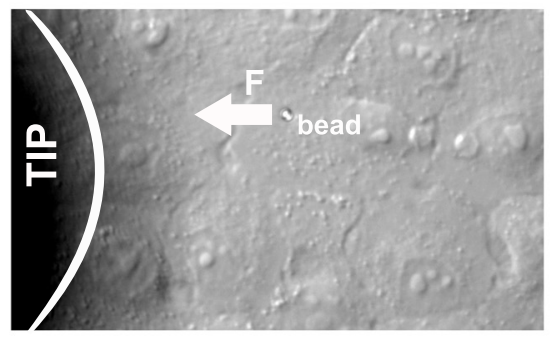

$\longrightarrow$ Ecad
$\rightarrow$ Pcad

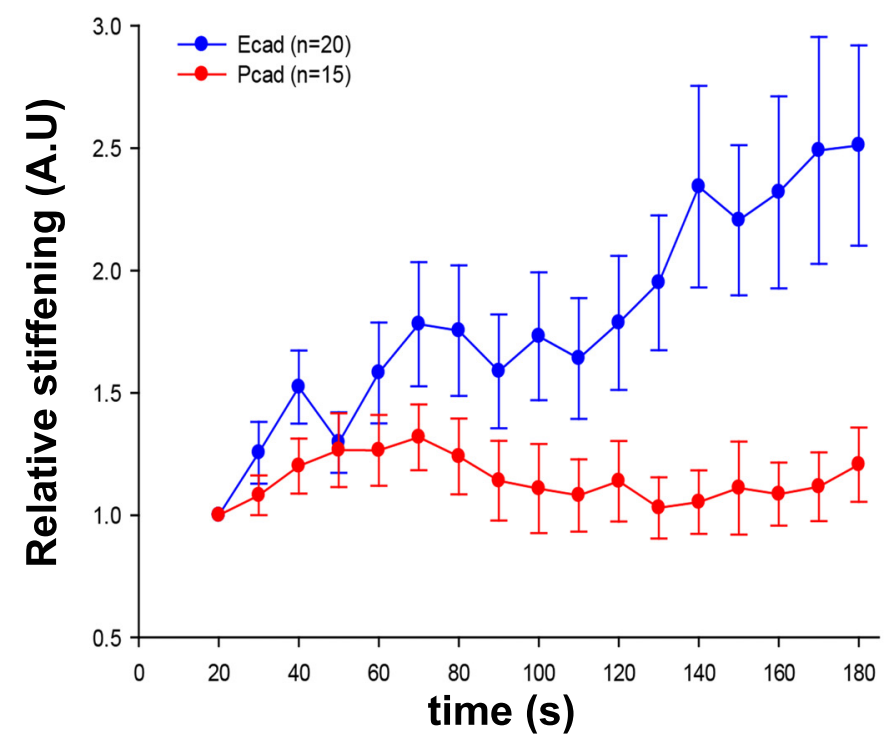

Fig 5 